\title{
Antimicrobial resistance in bacteria isolated from mastitis in dairy cattle in France, 2006-2016
}

\author{
Clémence Boireau, ${ }^{*} \dagger \ddagger$ Géraldine Cazeau, $\uparrow$ Nathalie Jarrige, $\uparrow$ Didier Calavas, $\uparrow$ Jean-Yves Madec, $\S$ \\ Agnès Leblond, $\ddagger$ Marisa Haenni, $§$ and Émilie Gay ${ }^{1}$ \\ *École Nationale des Services Vétérinaires (ENSV), Vetagro Sup, Marcy l'Étoile, 69280, France \\ †French Agency for Food, Environmental and Occupational Health and Safety (ANSES), Laboratoire de Lyon, Unité Épidémiologie, \\ Université de Lyon, 31 avenue Tony Garnier, 69007 Lyon, France \\ ‡Epidémiologie des maladies animales et zoonotiques, UMR 0346, INRA, Vetagro Sup, University of Lyon, F-69280, Marcy L'Etoile, France \\ §ANSES, Laboratoire de Lyon, Unité Antibiorésistance et Virulence Bactériennes, Université de Lyon, 31 avenue Tony Garnier, 69007 Lyon, \\ France
}

\begin{abstract}
In dairy cattle, mastitis is the most frequent bacterial disease, and the routine use of antibiotics for treatment and prevention can drive antimicrobial resistance (AMR). The aim of our study was to estimate the levels of AMR of the 3 main bacteria isolated from dairy cattle with mastitis in France (Streptococcus uberis, Escherichia coli, and coagulase-positive staphylococci) and to investigate their changes over time. Data collected between 2006 and 2016 by the French surveillance network for AMR in pathogenic bacteria of animal origin (called RESAPATH) were analyzed. The proportions of mono- and multidrug resistance were calculated and the trends were investigated using nonlinear analyses applied to time series. Over the whole period, the lowest proportions of resistance in S. uberis isolates were observed for oxacillin $(2.2 \%)$ and gentamicin (2.4\%) and most resistance levels were below 20\%. The trends in resistance showed some significant variation, mainly for $S$. uberis, but without a common pattern across the various antibiotics examined. For only 2 combinations of bacteria-antibiotic the trend in resistance showed a continuous increase from 2006 to 2016: tetracycline resistance in $S$. uberis isolates and third-generation cephalosporin resistance in E. coli isolates. In E. coli, the highest proportions of resistance were observed for amoxicillin (28.1\%) and tetracycline (23.1\%). Resistance to third-generation cephalosporins in E. coli from dairy cattle was almost nil in 2006, but reached $2.4 \%$ in December 2016. This increase is particularly concerning because these antibiotics constitute one of the latest therapeutic alternatives to fight severe infectious
\end{abstract}

Received April 5, 2018.

Accepted June 24, 2018.

${ }^{1}$ Corresponding author: emilie.gay@anses.fr diseases in humans. Except for penicillin (33.9\%), the proportions of resistance in coagulase-positive staphylococci were below $11 \%$ during the whole study period. Multidrug resistance (isolates with acquired resistance to at least one antibiotic in 3 or more antibiotic classes) ranged from $2.4 \%$ for coagulase-positive staphylococci to $9.9 \%$ for $S$. uberis. These findings can serve as guidelines for practitioners in the choice of the most appropriate antibiotic according to the prevailing epidemiological context. Ultimately, our results contribute to risk assessment of AMR and provide a baseline for setting up and evaluating control measures and designing strategies to limit AMR.

Key words: antimicrobial resistance, mastitis, time series, dairy cattle, RESAPATH

\section{INTRODUCTION}

Mastitis, the inflammation of the mammary gland, is the most frequent bacterial infection encountered in dairy cattle and is associated with reduced milk production and changes in milk composition. Dairy cattle mastitis is mainly caused by bacteria, and antibiotics are an important component of their treatment and control. In France (Guérin-Faublée et al., 2003; Botrel et al., 2010), as well as in other European, American, and African countries, Streptococcus uberis, Escherichia coli, and coagulase-positive staphylococci (CPS) are the most frequent causative pathogens of clinical mastitis (Tenhagen et al., 2006; Bradley et al., 2007; Ericsson Unnerstad et al., 2009; Kateete et al., 2013). For subclinical mastitis, E. coli appear to be less prevalent than CPS, CNS, and streptococci (Botrel et al., 2010; Persson et al., 2011). These 4 bacteria are of real concern for public health: they can be a reservoir for antimicrobial resistance (AMR) genes and can play a role in the spread of genes to other pathogenic and 
commensal bacteria in the farm environment (Aarestrup, 2015).

Because the use of antibiotics can lead to resistance (Aarestrup, 2015), AMR occurrence must be carefully monitored to guide prudent prescription (Thomas et al., 2015). Hundreds of publications describe the proportion of AMR in bovine mastitis pathogens in the last decades (Barlow, 2011), highlighting differences in AMR proportions between areas and populations (Erskine et al., 2002; Makovec and Ruegg, 2003; Pitkälä et al., 2004; Osterås et al., 2006; Tenhagen et al., 2006; Botrel et al., 2010; Idriss et al., 2014). But most studies are based on specific selected animal populations and represent point-in-time estimates of AMR in specific geographic regions: they do not provide an overall picture of the situation. In addition, AMR in cow mastitis has never been studied in Europe over a long (at least 4 yr) and continuous period of time to detect emergence and characterize AMR trends. However, the estimation of resistance levels and trends in udder pathogens is crucial to guide the appropriate prescription of antibiotics and thus preserve the therapeutic arsenal ensuring the long-term efficacy of antibiotics.

The aim of our study was to estimate the AMR levels in the 3 most frequent causative agents of mastitis in dairy cattle among those collected by the French national surveillance network for AMR, and to describe their variations from 2006 and 2016.

\section{MATERIALS AND METHODS}

\section{Source of Data}

This retrospective study was performed using data from the French established national surveillance network for AMR in pathogenic bacteria of animal origin, called RESAPATH. This network was created in 1982 and monitors AMR in all animal species. It is coordinated by the French Agency for Food, Environmental and Occupational Health and Safety (ANSES). RESAPATH collects antibiogram results from participating French veterinary laboratories $(74$ members in 2016 out of 112 in the country). These antibiograms are requested by veterinarians confronted with a clinical case of the pathology for diagnostic and treatment purposes.

The RESAPATH laboratories use standard bacteriological cultures and methods for bacterial isolation and identification. Even if each laboratory has its own strategy of bacterial identification, the biggest ones use mass spectrometers (Singhal et al., 2015). Antibiograms are performed using the disk diffusion method, according to recommendations of the Antibiogram Committee of the French Society of Microbiology (CA-SFM).
Inhibition zone diameters are communicated to ANSES and compiled in a database. In accordance with the breakpoints recommended by the veterinary section of the CA-SFM (2017), isolates are then categorized as susceptible, susceptible, or resistant. The RESAPATH laboratories have participated in annual quality assurance proficiency tests since 2006, which contributes to the quality control of the data.

From the RESAPATH database, we extracted data involving the 3 most frequent bacteria isolated from dairy cattle mastitis from 2006 to 2016, namely $S$. uberis, E. coli, and CPS. For CPS isolates, the information sent by several laboratories did not allow us to strictly distinguish between species so we chose to perform analysis on the category CPS. Variables extracted included bacterial species, sampling date, and susceptibility categories (susceptible, susceptible, or resistant) to each tested antibiotic. For the 3 bacteria considered, we selected antibiotics of relevance in veterinary and human medicine (Table 1).

\section{Data Analysis}

From an epidemiological point of view, the event of interest is the nonsusceptibility of the pathogen to the antibiotic tested, indicating that the isolated bacteria is no longer a wild-type strain. Therefore, intermediate isolates were grouped together with resistant isolates in the nonsusceptible bacterial population, referred to as resistant in this study. The indicator of AMR was defined for each bacteria and antibiotic as the proportion of the number of resistant isolates relative to the total number of isolates tested.

In a time series analysis, trends reflect structural dynamics and the detection of trends was used to monitor long-term movement, particularly in AMR (López-Lozano et al., 2000; Mühlemann et al., 2006; Doernberg et al., 2015; Boireau et al., 2018b). A trend is defined as the long-term variation along with seasonality and noise (i.e., the residual components). To compare our results with previous studies, we used generalized additive models (GAM; Dominici et al., 2002) to capture trends and seasonality. The time step was chosen according to the number of data available such that there were at least 25 antibiograms in each time step (Barlow, 2011) and to ensure the validity of the model (independent and normally distributed residuals); we used a bimonthly (every 2 mo) time step. Using count data, the bimonthly number of resistant isolates was modeled using a quasi-Poisson regression with an offset equal to the log of the total number of isolates submitted during the same period. The AMR trends were modeled for each bacterial species and ana- 
Table 1. Selected antibiotics and the corresponding antibiotic classes

\begin{tabular}{lll}
\hline Bacterial species & Antibiotic class & Antibiotic \\
\hline Streptococcus uberis & Penicillins & Oxacillin \\
& Aminoglycosides & Gentamicin \\
& Fluoroquinolones & Enrofloxacin \\
& Tetracyclines & Tetracycline \\
& Folate pathway inhibitors & Trimethoprim-sulfamethoxazole \\
& Macrolides & Erythromycin \\
Escherichia coli & Lincosamides & Lincomycin \\
& Penicillins & Amoxicillin \\
& Cephalosporins & Ceftiofur \\
& Aminoglycosides & Gentamicin \\
& Fluoroquinolones & Enrofloxacin \\
& Tetracyclines & Tetracycline \\
Coagulase-positive staphylococci & Folate pathway inhibitors & Trimethoprim-sulfamethoxazole \\
& Penicillins & Penicillin G \\
& Cephalosporins & Cefoxitin \\
& Aminoglycosides & Gentamicin \\
& Fluoroquinolones & Marbofloxacin \\
& Tetracyclines & Tetracycline \\
& Folate pathway inhibitors & Trimethoprim-sulfamethoxazole \\
& Macrolides & Erythromycin \\
& Lincosamides & Lincomycin \\
\hline
\end{tabular}

lyzed using a separate and independent GAM for each antibiotic. The models presented in this paper included nonparametric smooth functions of calendar time selected by cross-validation and designed to control for trend and seasonality (Wood, 2008). The GAM models were structured and analysis performed as described in Boireau et al. (2018b). Smoothing parameters were estimated using cross-validation. The adequacy of the models for the series was verified using white noise tests (Box-Ljung test, skewness-kurtosis, and Shapiro test) to check whether the residuals were independent and normally distributed. A $P$-value of $\leq 0.05$ was considered statistically significant. If trend variations were not significant, the trend was stationary. R ( R Core Team, 2017, R Foundation for Statistical Computing, Vienna, Austria), version 3.4.0. (2017-04-21), was used for all statistical analyses (gamm4 package for GAM implementation) and graphic illustrations.

Multidrug resistance analysis was carried out according to the definition proposed by Magiorakos et al. (2012). Isolates with acquired resistance to at least one antibiotic in 3 or more antibiotic classes (from those selected) were considered as multidrug resistant. The proportions of multi-susceptible isolates (susceptible to all classes of antibiotics selected for analysis), resistant to 1 or 2 antibiotic classes, multidrug-resistant, and resistant to all classes of antibiotics were calculated. Confidence intervals were calculated using the exact binomial method (2-sided $P$-values). These analyses were performed using 6 antibiotic classes for E. coli, 7 for $S$. uberis, and 8 classes for CPS (Table 1). Isolates with missing inhibition diameters to the aforementioned antibiotic classes were excluded.

\section{RESULTS}

A total of 27,888 antibiograms met the selection criteria (linked with mastitis in dairy cattle due to 1 of the 3 most frequent bacterial species isolated and including at least one of the selected antibiotics) in the RESAPATH database between January 1, 2006, and December 31, 2016. Among these, $49.7 \%$ of antibiograms involved S. uberis, $25.4 \%$ E. coli, and $24.9 \%$ CPS.

\section{Resistance Levels over the Whole Period}

Over the period, resistance levels varied between bacteria and antibiotics (Table 2). Resistance levels for S. uberis were low for oxacillin $(2.2 \%)$ and gentamicin $(2.4 \%)$, and attained $9.3 \%$ for trimethoprim-sulfamethoxazole (SXT), whereas resistance levels to tetracycline (18.1\%), lincomycin (19.1\%), and erythromycin (20.0\%) were around $20 \%$, and resistance to enrofloxacin reached $32.9 \%$. For E. coli and CPS isolates, levels of resistance to cephalosporins were low (1.4 and $7.9 \%$, respectively), but higher to penicillins (28.1 and $33.9 \%$, respectively). In contrast to $S$. uberis, the resistance levels to fluoroquinolones was close to zero for CPS $(0.7 \%)$ and low for $E$. coli $(2.6 \%)$. Resistance levels to SXT and gentamicin were below $10 \%$ for the 3 bacterial species studied (Table 2). In contrast, resistance to tetracycline differed between the 3 pathogens: low for 


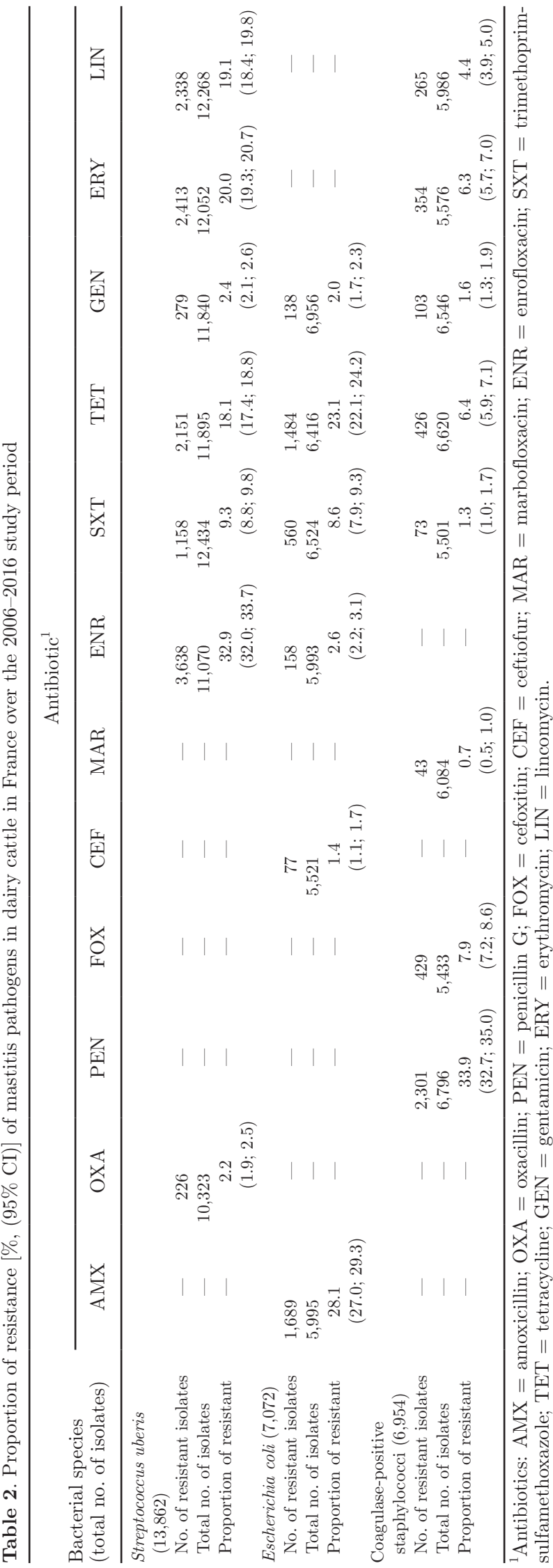

CPS (6.4\%), but around $20 \%$ for S. uberis $(18.1 \%)$ and for $E$. $\operatorname{coli}(23.1 \%)$.

\section{Antimicrobial Resistance Trends}

Based on graphical analyses, no seasonal cycle was observed and the seasonal component of the models was not significant for any of the pathogens studied (Supplemental Table S1; https://doi.org/10.3168/jds .2018-14835).

For S. uberis, significant $(P<0.05$ for all trends $)$ increasing AMR trends were demonstrated for all antibiotics during the second half of the study period (Figure 1). Resistance to tetracycline showed a linear increase from $15.7 \%(14.2 ; 17.2)$ in January 2006 to $20.4 \%(18.7 ; 22.1)$ in December 2016. In contrast, the trends were nonlinear for the other antibiotics tested. The proportion of resistance to oxacillin remained under $5 \%$ over the period: it declined slightly from 2006 to 2010 and then rapidly increased from $0.7 \%$ $(0.4 ; 1.0)$ in May 2010 up to $4.5 \%(3.1 ; 5.9)$ in July 2014 , and decreased again in 2015 and 2016. The proportion of resistance to gentamicin was always low: it decreased from $3.7 \%(1.9 ; 5.5)$ in January 2006 to $1.5 \%(1.1 ; 1.9)$ in January 2012 and then increased up to $3.4 \%(2.0 ; 4.7)$ in December 2016 . The proportion of resistance to lincomycin declined in the first half of the study period and then increased up to $22.4 \%$ $(20.2 ; 24.7)$ in July 2015 , before stabilizing. The proportion of resistance to SXT was stable around $6 \%$ from 2006 to 2012, increasing in January 2012 and reaching $16.0 \%(11.0 ; 21.0)$ in December 2016. Although the resistance to erythromycin was not stationary, the proportion of resistance varied little over the period. Finally, resistance to enrofloxacin slightly declined during the first half of the study period and then increased up to $41.8 \%(36.4 ; 47.2)$ in July 2015 , before decreasing again.

For E. coli, resistance trends to amoxicillin, enrofloxacin, SXT, and gentamicin were stationary from 2006 to 2016. In contrast, resistance to ceftiofur increased from $0.4 \%(0.0 ; 0.7)$ in January 2006 to $2.4 \%$ (1.5; 3.4) in December 2016 (Figure 2). Resistance to tetracycline showed large fluctuations over the study period, increasing from $19.5 \%(11.9 ; 27.2)$ in January 2006 up to a peak at $42.4 \%(37.0 ; 47.8)$ in July 2009 , before dropping to $15.7 \%(13.2 ; 18.2)$ in May 2012. Afterward, it rose slightly reaching $20.6 \%(16.1 ; 25.0)$ in December 2016.

For CPS, resistance trends to marbofloxacin, tetracycline, erythromycin, and gentamicin were stationary from 2006 to 2016. In contrast, the resistance trend to SXT was linear, decreasing from $2.1 \%(1.2 ; 3.1)$ in January 2006 to $0.8 \%(0.4 ; 1.2)$ in December 2016 (Fig- 

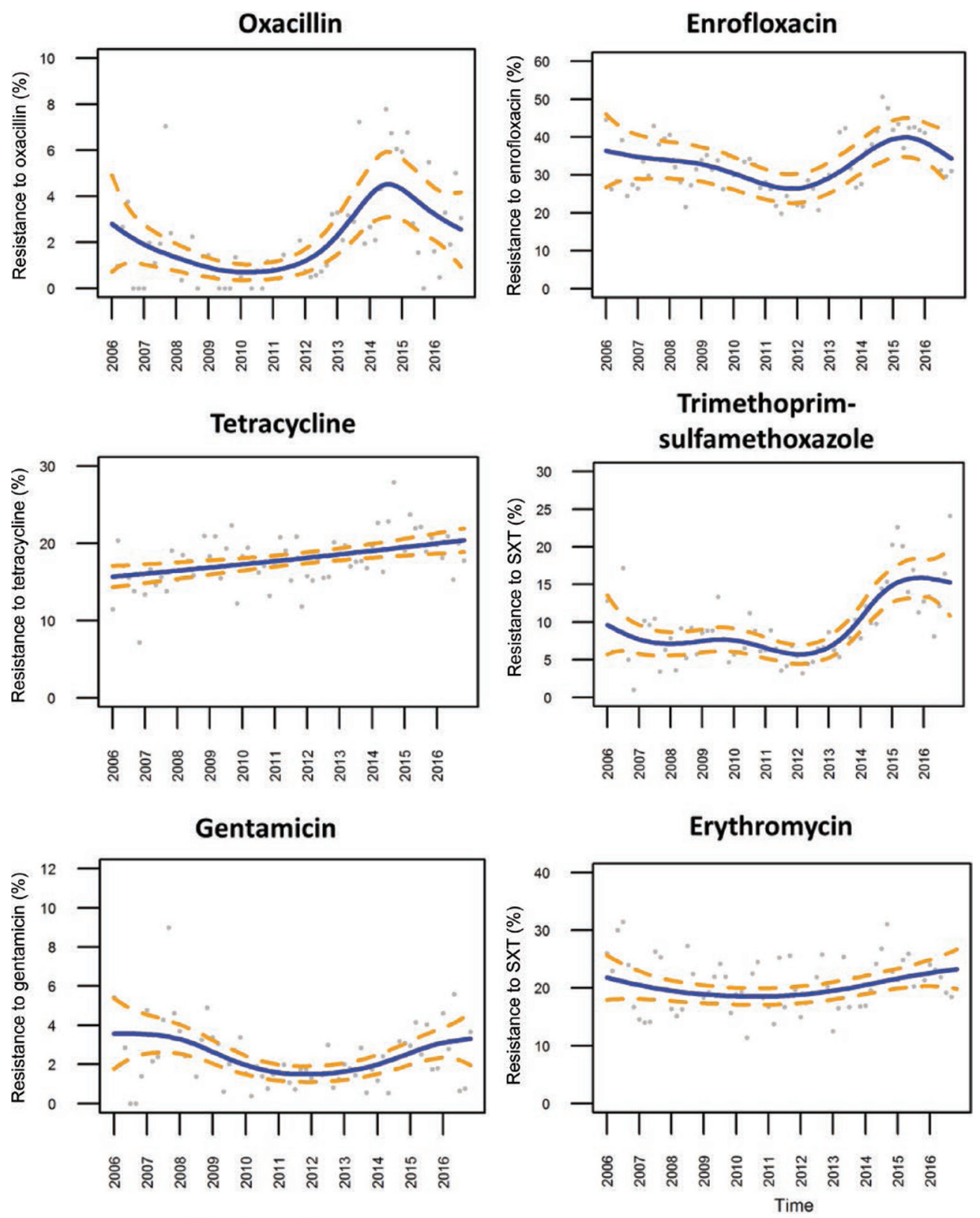

Lincomycin

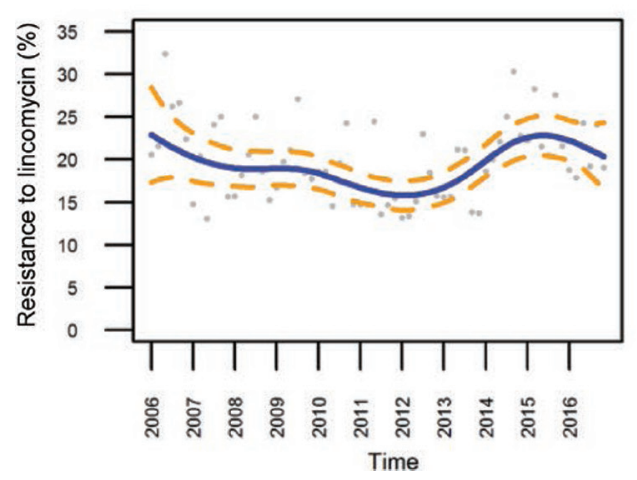

Figure 1. Trends (and 95\% CI; dashed lines) of antimicrobial resistance for Streptococcus uberis isolates from dairy cattle mastitis cases during the 2006-2016 period (bimonthly time step). Color version available online. 
ure 3). Similarly, the resistance trend to lincomycin was linear, decreasing from $5.8 \%(4.8 ; 6.8)$ in January 2006 to $3.5 \%(2.9 ; 4.0)$ in December 2016. The resistance to penicillin was primarily stable and then resistance proportions also decreased, from $39.1 \%$ (36.6; 41.7) in September 2008 to $23.4 \%(19.9 ; 26.9)$ in December 2016. Finally, the proportion of resistance to cefoxitin dropped from $27.6 \%(13.4 ; 41.9)$ in January 2006 to
Amoxicillin

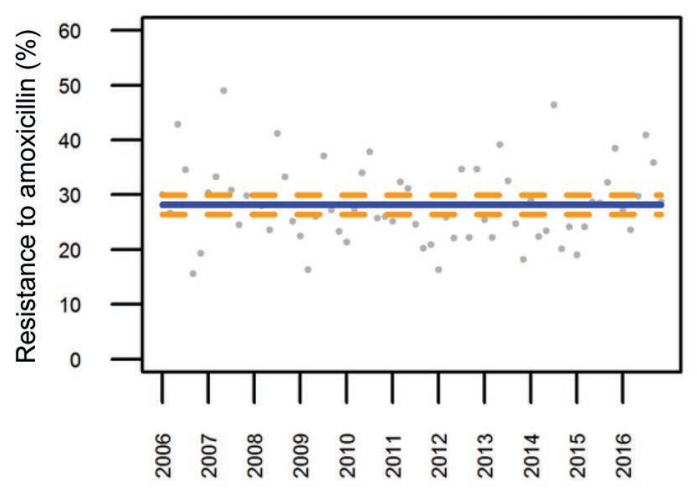

Tetracycline

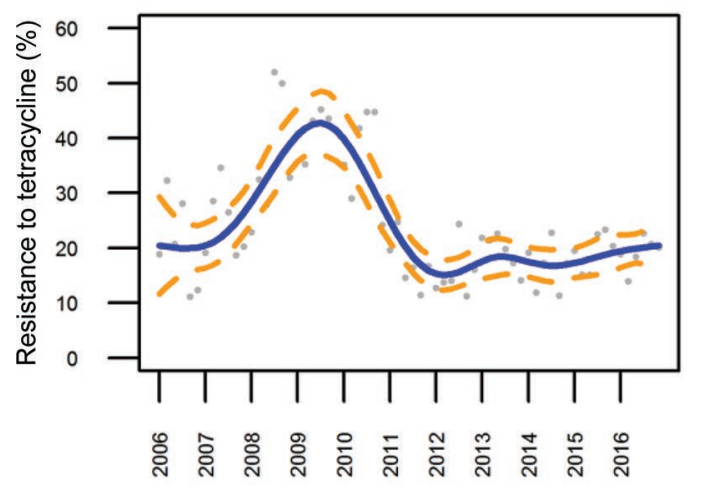

Gentamicin

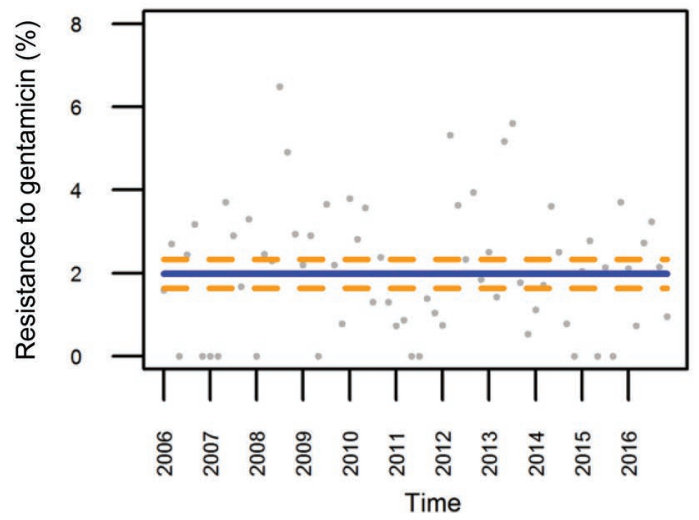

Enrofloxacin
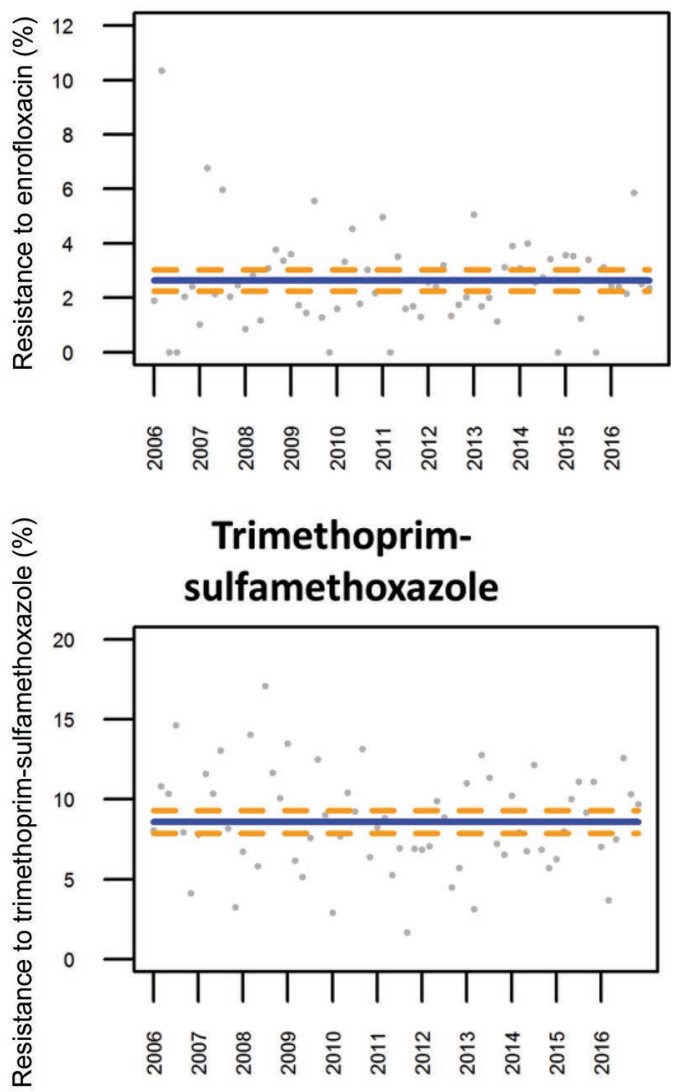

Ceftiofur

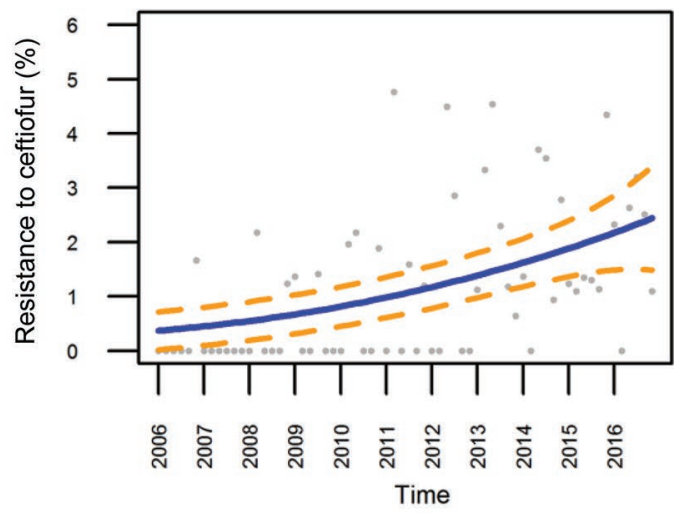

Figure 2. Trends (and 95\% CI; dashed lines) of antimicrobial resistance for Escherichia coli isolates from dairy cattle mastitis cases during the 2006-2016 period (bimonthly time step). Color version available online. 
Penicillin

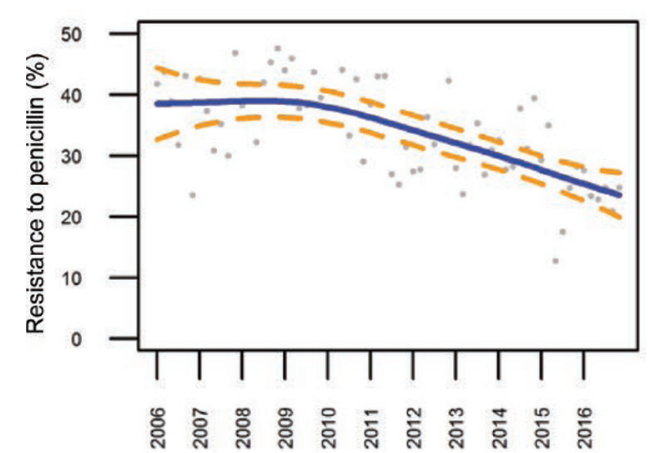

Tetracycline

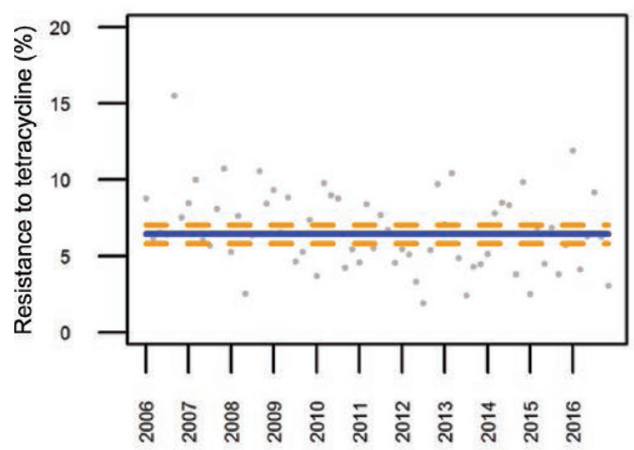

Gentamicin

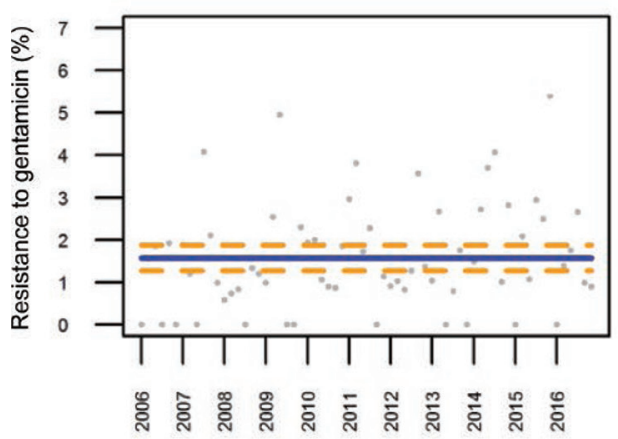

Cefoxitin

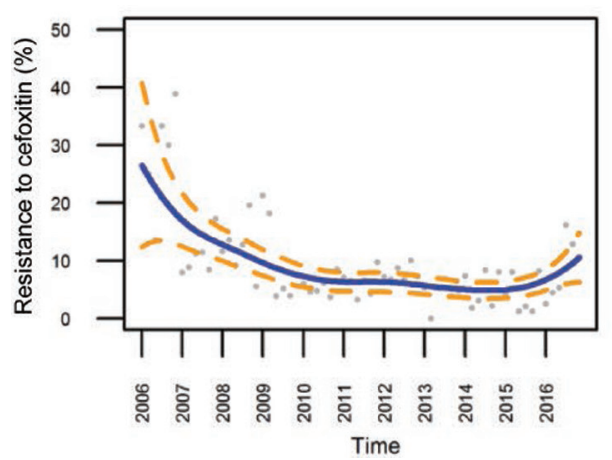

Marbofloxacin

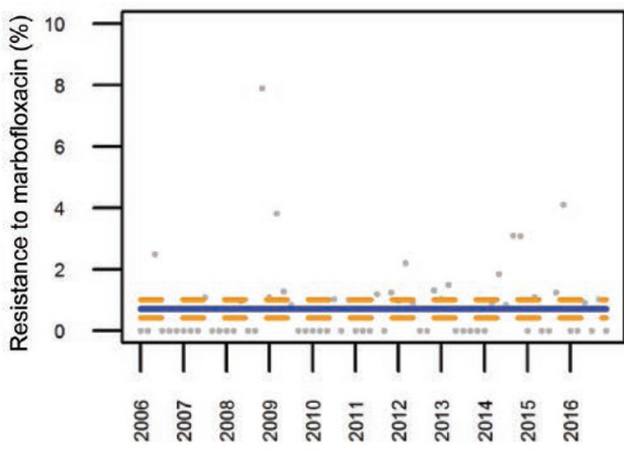

Trimethoprimsulfamethoxazole

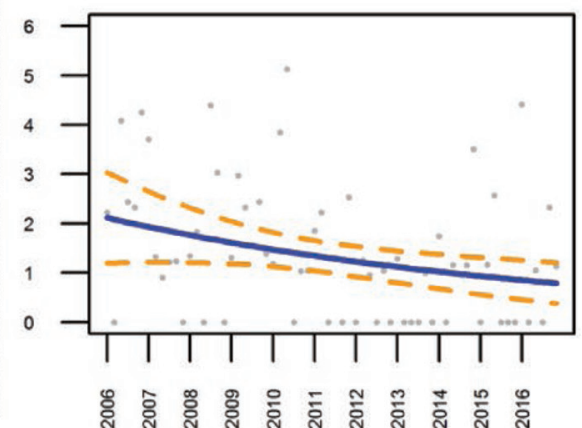

Erythromycin

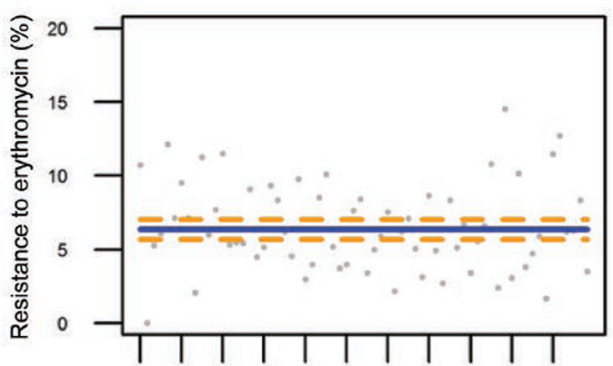

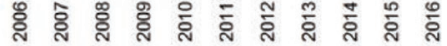

Lincomycin

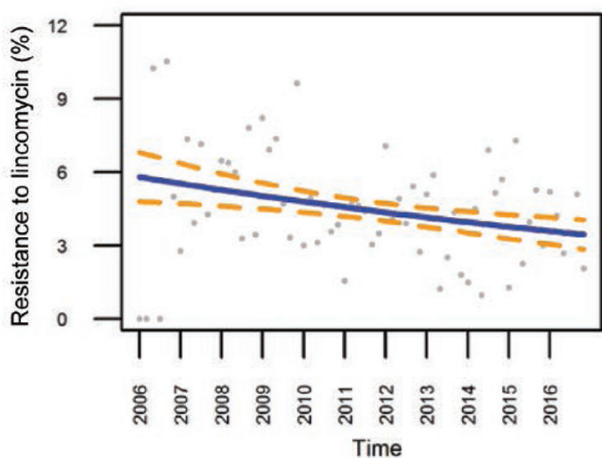

Figure 3. Trends (and 95\% CI; dashed lines) of antimicrobial resistance for coagulase-positive staphylococci isolates from dairy cattle mastitis cases during the 2006-2016 period (bimonthly time step). Color version available online. 
$4.7 \%(3.4 ; 6.1)$ in September 2014 and then increased up to $10.3 \%(5.8 ; 14.8)$ in December 2016.

\section{Multidrug Resistance}

The analysis included 13,436 isolates for which complete records on resistance to the aforementioned antibiotic classes were available (5,867 for $S$. uberis, 3,760 for E. coli, and 3,809 for CPS). For the 3 bacterial species studied, more than half of the isolates were susceptible to all classes of antibiotics considered (Table 3). Multidrug resistance proportions reached $3.6 \%$ for CPS, $7.6 \%$ for E. coli, and $14.5 \%$ for S. uberis. Only a few isolates were resistant to all classes considered.

\section{DISCUSSION}

\section{Antimicrobial Resistance in Bacteria Involved in Mastitis}

Here, we estimated the AMR of the 3 main bacteria isolated from mastitis cases in dairy cattle in France and its changes over time. To the best of our knowledge, this is the first study describing the resistance trends of udder pathogens using nonlinear time series analysis, even though the picture may be imperfect, considering the potential selection biases. Indeed, bacterial isolations and antibiograms are not systematically performed, relying on the decision taken by veterinarians during their clinical activity. Overall, these biases may lead to a lack of representativeness. However, providing that these biases do not vary over time, the resistance trends are meaningful. These findings also highlight the importance of considering both the level of resistance and any temporal variation to characterize the AMR and estimate its potential threat. Besides, a study regarding the RESAPATH network in 2015 concluded that the geographical coverage of the RESAPATH was broadly consistent with the geographical distribution of the estimated animal populations over the territory (Boireau et al., 2018a).

Antimicrobial resistance may vary between clinical and subclinical mastitis. Escherichia coli is an environmental pathogen strongly associated with severe clinical mastitis. However, for S. uberis and CPS, we could not distinguish between clinical and subclinical mastitis in this study, even if we assume that the vast majority of bacteria were isolated from clinical mastitis, because a history of clinical illness generally prompts sample submission by veterinarians. This assumption was also supported by the concordance between the distribution of pathogens collected in our study and the distribution of pathogens isolated from clinical mastitis in France in an earlier study (Botrel et al., 2010). In this study, we were also not able to distinguish between CPS species, because not all participating laboratories transferred the same detailed information concerning species identification for CPS. We know that among 6,954 CPS isolates at least 4,900 were Staph. aureus and we assume that the vast majority of the other isolates were Staph. aureus also as it was previously described in the literature (Bengtsson et al., 2009; Botrel et al., 2010). Considering the literature and that Staph. aureus was always distantly the first species isolated by laboratories transferring the full species identification, we also assume that if a change in species had occurred over the period, it had minimal effect on the results of this study, even if their accuracy might be affected.

Compared with monoresistance analyses, the multidrug resistance estimates were performed on a limited number of isolates, especially for $S$. uberis, because many records on resistance to the selected antibiotics were incomplete, due to nonsystematic testing across antibiotic panels for a given bacteria at veterinary

Table 3. Proportions [\%, (95\% CI)] of isolates that were multi-susceptible, resistant to 1 or 2 antibiotic classes, multidrug-resistant, and resistant to all classes of antibiotics selected for analysis over the 2006-2016 period

\begin{tabular}{|c|c|c|c|}
\hline $\begin{array}{l}\text { Bacterial species } \\
\text { (total number of isolates) }\end{array}$ & Susceptibility patterns of isolates & $\begin{array}{l}\text { No. of } \\
\text { isolates }\end{array}$ & $\begin{array}{l}\text { Proportion } \\
(95 \% \text { CI })\end{array}$ \\
\hline \multirow[t]{3}{*}{ Streptococcus uberis $(5,867)$} & Multi-susceptible & 3,105 & $52.9(51.6 ; 54.2)$ \\
\hline & Resistant to 1 or 2 antibiotic classes & 1,911 & $32.6(31.4 ; 33.8)$ \\
\hline & Resistant to all classes of antibiotics selected & 28 & $0.5(0.3 ; 0.7)$ \\
\hline \multirow[t]{2}{*}{ Escherichia coli $(3,760)$} & Multi-susceptible & 2,580 & $68.6(67.1 ; 70.1)$ \\
\hline & Resistant to 1 or 2 antibiotic classes & 896 & $23.8(22.5 ; 25.2)$ \\
\hline \multirow{4}{*}{$\begin{array}{l}\text { Coagulase-positive } \\
\text { staphylococci }(3,809)\end{array}$} & Multi-susceptible & 2,362 & $62.0(60.4 ; 63.6)$ \\
\hline & Resistant to 1 or 2 antibiotic classes & 1,311 & $34.4(32.9 ; 36.0)$ \\
\hline & Multidrug-resistant & 136 & $3.6(3.0 ; 4.2)$ \\
\hline & Resistant to all classes of antibiotics selected & 3 & $0.1(0.0 ; 0.2)$ \\
\hline
\end{tabular}


laboratories. Nevertheless, our study showed that resistance levels varied greatly among antibiotic classes. Unfortunately, the limited number of isolates prevented modeling the proportions of multidrug resistance over time. From a surveillance point of view, there is clearly a need to harmonize practices between laboratories at the national level to help improve the surveillance of AMR in France.

To properly compare results of AMR between studies, various elements need to be considered: the sample selection and size, the design of isolate collection, the bacterial population studied, the time and place of the study, the methods of susceptibility testing performed, the breakpoints and references used, and the analyses carried out. Meeting all these criteria make data comparison difficult.

The resistance proportions in streptococci isolated from mastitis have rarely been assessed in the literature due to the lack of available breakpoints (Tenhagen et al., 2006; Persson et al., 2011). For S. uberis, the highest level of resistance was observed for enrofloxacin (32.9\%) in our study. However, we very likely overestimated the resistance to fluoroquinolones in streptococci isolates because we grouped intermediate and resistant isolates. Due to their intrinsic resistance to quinolones, streptococci isolates may appear phenotypically intermediate using the disk diffusion method, despite the lack of any acquired resistance. We observed that resistance to oxacillin was low for S. uberis from 2006 to 2016 (2.2\%), whereas a previous French study conducted in 2007 and 2008 in a single French region reported a much higher level of resistance (12.6\%; Botrel et al., 2010). This difference is most likely due to the change in breakpoint recommendations for this bacteria-antibiotic combination in 2010. In this same study, the authors reported lower levels of resistance to tetracycline $(7.2 \%)$ than ours (18.1\%); this may also be due to differences in breakpoints used and a heterogeneous spatial distribution of AMR in mastitis, potentially linked to a heterogeneous spatial distribution of practices and farm management. Recently, a European study performed on 282 S. uberis isolates from dairy cattle with clinical mastitis found results similar to ours for erythromycin resistance (20.0\%), but they identified higher resistance to tetracycline $28.7 \%$ using the CLSI breakpoint; Thomas et al., 2015).

For E. coli., the resistance to tetracycline (23.1\%) over the whole study period was similar to results obtained in the Netherlands, Switzerland, and Belgium (Hendriksen et al., 2008; Supré et al., 2014). In comparison with a previous French study (Botrel et al., 2010; data from 2007 to 2008), we systematically observed higher resistance levels for amoxicillin (28.1 vs. 9.7\%), tetracycline (23.1 vs. $10.4 \%)$, and gentamicin resistance (2.0 vs. $0.7 \%)$. As mentioned above, these differences may be due to the spatial sampling, suggesting potential difference in antibiotics use or practices between French regions. Previous studies based on the disk diffusion method have observed very low or nil resistance to ceftiofur in European countries and France (Hendriksen et al., 2008; Bengtsson et al., 2009; Botrel et al., 2010; Idriss et al., 2014; Thomas et al., 2015). Our findings especially showed an increase in resistance to third-generation cephalosporins (3GC) in E. coli collected from dairy cattle. Even if the amplitude is limited, this rise is particularly worrying because 3GC have been classified as critically important antibiotics (Collignon et al., 2009). In comparison with other animal production sectors, a previous study reported that after reaching high levels of resistance, trends in 3GC resistance have been declining in poultry and swine production since 2010 and in 2015 their proportions of resistance were below 10\% (Boireau et al., 2018b). Resistance dynamics are clearly different between animal species and pathologies. In cattle (across all pathology and livestock production sectors), Boireau et al. (2018b) similarly reported low but increasing resistance from 2002 to 2014, with a stabilizing trend since 2014. Here, in contrast, our results showed that $3 \mathrm{GC}$ resistance in dairy cattle with mastitis has been continuously increasing. The dynamics of $3 \mathrm{GC}$ resistance observed in the present study underscores the importance of focusing studies on specific production sectors or particular pathologies (or both) to fine-tune the characterization of AMR and detect changes.

The proportions of resistance for CPS isolates in the present study are consistent with previous French results (Botrel et al., 2010; data from 2007 to 2008), except for higher resistance to penicillin (33.9 vs. $14.1 \%)$. However, more recent studies indicate similar levels of resistance in France $37.5 \%$; Thomas et al., 2015). Changes over time may explain these differences between studies; our results confirmed that penicillin resistance varied from 2006 to 2016, but this variation cannot be the only explanation for the discrepancies between estimates. Although most resistance trends in CPS were stationary, resistance to penicillin, SXT and cefoxitin (marker for methicillin resistance) varied over the period. We can speculate that the observed changes in proportion of resistance might be related to a shift in Staph. aureus strains over the period (Sakwinska et al., 2011). Due to a lack of species identification for CPS and a lack of further genetic investigations (for example, mecA-specific detection by PCR), we may 
have overestimated the actual proportion of methicillinresistant Staph. aureus (MRSA). Nevertheless, even if this is an overestimate of the real proportion of MRSA, we can consider that the bias is constant over time, providing for a meaningful trend. The proportion of phenotypically MRSA decreased rapidly in the first half of the period, but increased from 4.7 to $10.3 \%$ in the recent years, which is cause for concern.

\section{Consequences for Antimicrobial Therapy}

According to antibiotics sales records, penicillins, cephalosporins (first and second generations), aminoglycosides, polymyxins, and tetracyclines are the antibiotic classes the most frequently used for intramammary infusion in France (ANSES-ANMV, 2016). Moreover, the sales of last-generation cephalosporins for intramammary infusion increased by $20.3 \%$ between 2007 and 2011 and then decreased by $79.5 \%$ between 2013 and 2016, due to new regulations restraining their use in veterinary medicine and the increasing awareness of professionals (ANSES-ANMV, 2017). Considering the trend for $3 \mathrm{GC}$ resistance, we can suppose that the use of last-generation cephalosporins likely have selected for resistant bacteria, leading to $E$. coli mastitis more frequently caused by 3GC-resistant isolates, that 3GC-resistant E. coli isolates are more frequent in the environment, or both.

Among the 29 intramammary infusions available in France in 2017, 15 contained penicillins, 8 contained aminoglycosides, 6 contained first-generation cephalosporins, and 2 contained last-generation cephalosporins (one with cefoperazone and another with cefquinome). Our findings characterized AMR to all antibiotic classes available on the French market, except polymyxins and ansamycins. The latter class is not routinely tested by veterinary laboratories and we could not investigate its resistance. We were not able to analyze AMR to polymyxins because the disk diffusion method used by the RESAPATH laboratories cannot clearly discriminate susceptible and resistant isolates due to poor diffusion of polypeptide antibiotics in agar (Humphries, 2015).

Our findings can assist French veterinarians in their selection of the most appropriate antimicrobial agent for initial empirical therapy. They can also supplement therapeutic guidelines (SNGTV, 2014), which steer the therapeutic strategy in a given clinical and epidemiological context. Thus our results are part of comprehensive strategy to fight AMR (CGAAER, 2016). However, the control of mastitis cannot be reduced to antimicrobial susceptibility testing and prudent antibiotic use. Mastitis control and prevention is first based on (1) the identification of the mechanisms of infec- tion, with the definition of the clinical and subclinical states of the disease, (2) the discovery of appropriate screening tests, (3) the determination of likely points of exposure, and (4) the identification of pathogen-specific characteristics, such as AMR (Ruegg, 2017). Most of all, proper biosecurity, improved hygiene (postmilking teat dipping, hygienic milking procedures), and minimizing exposure to antibiotics may be important factors to reduce the burden of resistant bacteria on dairy farms.

\section{CONCLUSIONS}

This study provides a consistent source of data on AMR levels and trends of mastitis pathogens in French dairy cattle. Using time-series analyses, we detected trends in this complex and multifactorial phenomenon from 2006 to 2016. Despite resistance variations during the study period, we showed that for the vast majority of combinations of bacteria-antibiotic considered, antimicrobial resistance has not globally increased in mastitis pathogens from 2006 to 2016. The usefulness of monitoring programs such as RESAPATH to guide therapeutic practices, design control strategies, and detect emergence is substantial: there is clearly a need to set up national surveillance networks that can monitor AMR of pathogens isolated in dairy cattle and more generally in food-producing animals and pets. Overall, our results contribute to assessing the risk of AMR and provide an objective baseline to test the efficacy of AMR control measures. Ultimately, the knowledge of AMR trends in mastitis pathogens promotes the responsible use of antibiotics, which is required to curb AMR.

\section{ACKNOWLEDGMENTS}

The authors thank all the RESAPATH laboratories that have been collecting and transmitting antibiogram results for several years. The authors are particularly grateful to Christelle Philippon (RESAPATH secretary) for her meticulous follow-up of laboratories and careful data collection and Jean-Luc Vinard (RESAPATH data architect) for his careful management of the database. This work was partly supported by the French Ministry of Agriculture (http://agriculture.gouv.fr). No additional external funding was received for this study. The funders had no role in study design, data collection and analysis, decision to publish, or preparation of the manuscript. The authors declare no potential conflicts of interest with respect to the research, authorship, or publication of this article. The data used for this study was obtained from the RESAPATH network. Conditions of approval (respecting the anonymity of farms 
and laboratories) do not allow us to distribute or make data available directly to other parties. All the authors read and approved the final manuscript.

\section{REFERENCES}

Aarestrup, F. M. 2015. The livestock reservoir for antimicrobial resistance: A personal view on changing patterns of risks, effects of interventions and the way forward. Philos. Trans. R. Soc. Lond. B Biol. Sci. 370:20140085. https://doi.org/10.1098/rstb.2014.0085.

ANSES-ANMV. 2016. Suivi des ventes de médicaments vétérinaires contenant des antibiotiques en France en 2015. ANSES, Lyon, France.

ANSES-ANMV. 2017. Suivi des ventes de médicaments vétérinaires contenant des antibiotiques en France en 2016. ANSES, Lyon, France.

Barlow, J. 2011. Mastitis therapy and antimicrobial susceptibility: A multispecies review with a focus on antibiotic treatment of mastitis in dairy cattle. J. Mammary Gland Biol. Neoplasia 16:383-407. https://doi.org/10.1007/s10911-011-9235-z.

Bengtsson, B., H. E. Unnerstad, T. Ekman, K. Artursson, M. NilssonOst, and K. P. Waller. 2009. Antimicrobial susceptibility of udder pathogens from cases of acute clinical mastitis in dairy cows. Vet. Microbiol. 136:142-149. https://doi.org/10.1016/j.vetmic.2008.10 .024 .

Boireau, C., N. Jarrige, G. Cazeau, É. Jouy, M. Haenni, C. Philippon, D. Calavas, J.-Y. Madec, A. Leblond, and E. Gay. 2018a. Représentativité et couverture du résapath, le réseau d'épidémiosurveillance de l'antibiorésistance des bactéries pathogènes animales. Bull. Épidémiologique Santé Anim. Aliment. 80:10-14.

Boireau, C., É. Morignat, G. Cazeau, N. Jarrige, É. Jouy, M. Haenni, J.-Y. Madec, A. Leblond, and E. Gay. 2018b. Antimicrobial resistance trends in Escherichia coli isolated from diseased foodproducing animals in France: A 14-year period time-series study. Zoonoses Public Health 65:e86-e94. https://doi.org/10.1111/zph .12412 .

Botrel, M.-A., M. Haenni, E. Morignat, P. Sulpice, J.-Y. Madec, and D. Calavas. 2010. Distribution and antimicrobial resistance of clinical and subclinical mastitis pathogens in dairy cows in RhôneAlpes, France. Foodborne Pathog. Dis. 7:479-487. https://doi.org/ 10.1089/fpd.2009.0425.

Bradley, A. J., K. A. Leach, J. E. Breen, L. E. Green, and M. J. Green. 2007. Survey of the incidence and aetiology of mastitis on dairy farms in England and Wales. Vet. Rec. 160:253-257.

CA-SFM. 2017. Recommandations 2017 du comité de l'antibiogramme de la Société Française de Microbiologie, Groupe de Travail Antibiogramme Vétérinaire.

CGAAER. 2016. Le plan écoantibio 2012-2016: Evaluation, Recommandations pour le plan suivant. Conseil général de l'alimentation de l'agriculture et des espaces ruraux (CGAAER). MAAF, Paris, France.

Collignon, P., J. H. Powers, T. M. Chiller, A. Aidara-Kane, and F. M. Aarestrup. 2009. World Health Organization ranking of antimicrobials according to their importance in human medicine: A critical step for developing risk management strategies for the use of antimicrobials in food production animals. Clin. Infect. Dis. 49:132-141. https://doi.org/10.1086/599374.

Doernberg, S. B., V. Dudas, and K. K. Trivedi. 2015. Implementation of an antimicrobial stewardship program targeting residents with urinary tract infections in three community long-term care facilities: A quasi-experimental study using time-series analysis. Antimicrob. Resist. Infect. Control 4:54. https://doi.org/10.1186/ s13756-015-0095-y.

Dominici, F., A. McDermott, S. L. Zeger, and J. M. Samet. 2002. On the use of generalized additive models in time-series studies of air pollution and health. Am. J. Epidemiol. 156:193-203.

Ericsson Unnerstad, H., A. Lindberg, K. Persson Waller, T. Ekman, K. Artursson, M. Nilsson-Ost, and B. Bengtsson. 2009. Microbial aetiology of acute clinical mastitis and agent-specific risk factors. Vet. Microbiol. 137:90-97. https://doi.org/10.1016/j.vetmic.2008 .12 .005 .

Erskine, R. J., R. D. Walker, C. A. Bolin, P. C. Bartlett, and D. G. White. 2002. Trends in antibacterial susceptibility of mastitis pathogens during a seven-year period. J. Dairy Sci. 85:1111-1118. https://doi.org/10.3168/jds.S0022-0302(02)74172-6.

Guérin-Faublée, V., G. Carret, and P. Houffschmitt. 2003. In vitro activity of 10 antimicrobial agents against bacteria isolated from cows with clinical mastitis. Vet. Rec. 152:466-471.

Hendriksen, R. S., D. J. Mevius, A. Schroeter, C. Teale, D. Meunier, P. Butaye, A. Franco, A. Utinane, A. Amado, M. Moreno, C. Greko, K. Stärk, C. Berghold, A.-L. Myllyniemi, D. Wasyl, M. Sunde, and F. M. Aarestrup. 2008. Prevalence of antimicrobial resistance among bacterial pathogens isolated from cattle in different European countries: 2002-2004. Acta Vet. Scand. 50:28. https://doi .org/10.1186/1751-0147-50-28.

Humphries, R. M. 2015. Susceptibility testing of the polymyxins: Where are we now? Pharmacotherapy 35:22-27. https://doi.org/ 10.1002/phar.1505.

Idriss, S. E., V. Foltys, V. Tancin, K. Kirchnerova, D. Tancinova, and K. Zaujec. 2014. Mastitis pathogens and their resistance against antimicrobial agents in dairy cows in Nitra, Slovakia. Slovak J. Anim. Sci. 47:33-38.

Kateete, D. P., U. Kabugo, H. Baluku, L. Nyakarahuka, S. Kyobe, M. Okee, C. F. Najjuka, and M. L. Joloba. 2013. Prevalence and antimicrobial susceptibility patterns of bacteria from milkmen and cows with clinical mastitis in and around Kampala, Uganda. PLoS One 8:e63413. https://doi.org/10.1371/journal.pone.0063413.

López-Lozano, J. M., D. L. Monnet, A. Yagüe, A. Burgos, N. Gonzalo, P. Campillos, and M. Saez. 2000. Modelling and forecasting antimicrobial resistance and its dynamic relationship to antimicrobial use: A time series analysis. Int. J. Antimicrob. Agents 14:21-31.

Magiorakos, A.-P., A. Srinivasan, R. B. Carey, Y. Carmeli, M. E. Falagas, C. G. Giske, S. Harbarth, J. F. Hindler, G. Kahlmeter, B. Olsson-Liljequist, D. L. Paterson, L. B. Rice, J. Stelling, M. J. Struelens, A. Vatopoulos, J. T. Weber, and D. L. Monnet. 2012. Multidrug-resistant, extensively drug-resistant and pandrug-resistant bacteria: An international expert proposal for interim standard definitions for acquired resistance. Clin. Microbiol. Infect. 18:268-281. https://doi.org/10.1111/j.1469-0691.2011.03570.x.

Makovec, J. A., and P. L. Ruegg. 2003. Antimicrobial resistance of bacteria isolated from dairy cow milk samples submitted for bacterial culture: 8,905 samples (1994-2001). J. Am. Vet. Med. Assoc. $222: 1582-1589$.

Mühlemann, K., D. E. Uehlinger, W. Büchi, M. Gorgievski, and C. Aebi. 2006. The prevalence of penicillin-non-susceptible Streptococcus pneumoniae among children aged $<5$ years correlates with the biannual epidemic activity of respiratory syncytial virus. Clin. Microbiol. Infect. 12:873-879. https://doi.org/10.1111/j.1469-0691 .2006.1472_1.x.

Osterås, O., L. Sølverød, and O. Reksen. 2006. Milk culture results in a large Norwegian survey-Effects of season, parity, days in milk, resistance, and clustering. J. Dairy Sci. 89:1010-1023. https://doi .org/10.3168/jds.S0022-0302(06)72167-1.

Persson, Y., A.-K. J. Nyman, and U. Grönlund-Andersson. 2011. Etiology and antimicrobial susceptibility of udder pathogens from cases of subclinical mastitis in dairy cows in Sweden. Acta Vet. Scand. 53:36. https://doi.org/10.1186/1751-0147-53-36.

Pitkälä, A., M. Haveri, S. Pyörälä, V. Myllys, and T. Honkanen-Buzalski. 2004. Bovine mastitis in Finland 2001-Prevalence, distribution of bacteria, and antimicrobial resistance. J. Dairy Sci. 87:24332441. https://doi.org/10.3168/jds.S0022-0302(04)73366-4.

Ruegg, P. L. 2017. A 100-Year Review: Mastitis detection, management, and prevention. J. Dairy Sci. 100:10381-10397. https://doi .org/10.3168/jds.2017-13023.

Sakwinska, O., M. Giddey, M. Moreillon, D. Morisset, A. Waldvogel, and P. Moreillon. 2011. Staphylococcus aureus host range and human-bovine host shift. Appl. Environ. Microbiol. 77:5908-5915. https://doi.org/10.1128/AEM.00238-11. 
Singhal, N., M. Kumar, P. K. Kanaujia, and J. S. Virdi. 2015. MALDITOF mass spectrometry: An emerging technology for microbial identification and diagnosis. Front. Microbiol. 6:791. https://doi .org/10.3389/fmicb.2015.00791.

SNGTV. 2014. Recommandations de bonnes pratiques d'utilisation des antibiotiques en filière bovine. Société Nationale des Groupements Techniques Vétérinaires (SNGTV), Paris, France.

Supré, K., K. Lommelen, and L. De Meulemeester. 2014. Antimicrobial susceptibility and distribution of inhibition zone diameters of bovine mastitis pathogens in Flanders, Belgium. Vet. Microbiol. 171:374-381. https://doi.org/10.1016/j.vetmic.2014.02.045.

Tenhagen, B.-A., G. Köster, J. Wallmann, and W. Heuwieser. 2006. Prevalence of mastitis pathogens and their resistance against antimicrobial agents in dairy cows in Brandenburg, Germany. J. Dairy Sci. 89:2542-2551. https://doi.org/10.3168/jds.S0022 -0302(06)72330-X.

Thomas, V., A. de Jong, H. Moyaert, S. Simjee, F. El Garch, I. Morrissey, H. Marion, and M. Vallé. 2015. Antimicrobial susceptibility monitoring of mastitis pathogens isolated from acute cases of clinical mastitis in dairy cows across Europe: VetPath results. Int. J. Antimicrob. Agents 46:13-20. https://doi.org/10.1016/j .ijantimicag.2015.03.013.

Wood, S. N. 2008. Fast stable direct fitting and smoothness selection for generalized additive models. J. R. Stat. Soc. Ser. B Stat. Methodol. 70:495-518. https://doi.org/10.1111/j.1467-9868.2007 .00646.x. 